\title{
ON DECOMPOSING REGULAR GRAPHS INTO ISOMORPHIC DOUBLE-STARS
}

\author{
SaAd I. El-Zanati, Marie Ermete \\ James Hasty, Michael J. Plantholt
}

AND

\author{
Shailesh Tipnis \\ Department of Mathematics \\ Illinois State University \\ Normal, Illinois 61790-4520, U.S.A. \\ e-mail: saad@ilstu.edu \\ ermet1mn@gmail.com \\ HastyJ@bismarck.k12.il.us \\ mikep@ilstu.edu \\ tipnis@ilstu.edu
}

\begin{abstract}
A double-star is a tree with exactly two vertices of degree greater than 1 . If $T$ is a double-star where the two vertices of degree greater than one have degrees $k_{1}+1$ and $k_{2}+1$, then $T$ is denoted by $S_{k_{1}, k_{2}}$. In this note, we show that every double-star with $n$ edges decomposes every $2 n$-regular graph. We also show that the double-star $S_{k, k-1}$ decomposes every $2 k$-regular graph that contains a perfect matching.
\end{abstract}

Keywords: graph decomposition, double-stars.

2010 Mathematics Subject Classification: 05C51, 05C05.

\section{REFERENCES}

[1] P. Adams, D. Bryant and M. Buchanan, A survey on the existence of G-designs, J. Combin. Des. 16 (2008) 373-410.

doi:10.1002/jcd.20170

[2] D. Bryant and S. El-Zanati, Graph decompositions, in: Handbook of Combinatorial Designs, C.J. Colbourn and J.H. Dinitz (Ed(s)), (2nd Ed., Chapman \& Hall/CRC, Boca Raton, 2007) 477-485. 
[3] S.I. El-Zanati, M.J. Plantholt and S. Tipnis, On decomposing even regular multigraphs into small isomorphic trees, Discrete Math. 325 (2014) 47-51. doi:10.1016/j.disc.2014.02.011

[4] J.A Gallian, A dynamic survey of graph labeling, Electron. J. Combin. 16 (2013) \#DS6.

[5] R. Häggkvist, Decompositions of complete bipartite graphs, London Math. Soc. Lecture Note Ser. C.U.P., Cambridge 141 (1989) 115-147.

[6] M.S. Jacobson, M. Truszczyński and Zs. Tuza, Decompositions of regular bipartite graphs, Discrete Math. 89 (1991) 17-27. doi:10.1016/0012-365X(91)90396-J

[7] F. Jaeger, C. Payan and M. Kouider, Partition of odd regular graphs into bistars, Discrete Math. 46 (1983) 93-94. doi:10.1016/0012-365X(83)90275-3

[8] K.F. Jao, A.V. Kostochka and D.B. West, Decomposition of Cartesian products of regular graphs into isomorphic trees, J. Comb. 4 (2013) 469-490.

[9] A. Kotzig, Problem 1, in: Problem session, Proceedings of the Tenth Southeastern Conference on Combinatorics, Graph Theory and Computing, Congr. Numer. XXIV (1979) 913-915.

[10] G. Ringel, Problem 25, in: Theory of Graphs and its Applications, Proc. Symposium Smolenice 1963, Prague (1964), 162.

[11] H. Snevily, Combinatorics of Finite Sets, Ph.D. Thesis, (University of Illinois 1991).

Received 26 August 2013

Revised 7 February 2014

Accepted 10 February 2014 